\title{
EMR: A NEW METRIC TO ASSESS THE RESILIENCE OF DIRECTIONAL MMWAVE CHANNELS TO BLOCKAGES
}

\author{
Fatih Erden ${ }^{1}$, Ozgur Ozdemir ${ }^{1}$, Ismail Guvenc ${ }^{1}$, David W. Matolak ${ }^{2}$ \\ ${ }^{1}$ Department of Electrical and Computer Engineering, NC State University, Raleigh, NC 27606, ${ }^{2}$ Department of Electrical \\ Engineering, University of South Carolina, Columbia, SC 29208 \\ NOTE: Corresponding author: Fatih Erden, ferden@ncsu.edu
}

\begin{abstract}
Millimeter-wave (mmWave) communication systems require narrow beams to compensate for high path loss and to increase the communication range. If an obstacle blocks the dominant communication direction, alternative paths (directions) should be quickly identified to maintain reliable connectivity. In this paper, we introduce a new metric to quantify the Effective Multipath Richness (EMR) of a directional communication channel in the angular domain. In particular, the proposed metric takes into account the strength and spatial diversity of the resolved Multipath Components (MPCs), while also considering the beamwidth of the communication link and the blockage characteristics. The metric is defined as a weighted sum of the number of distinct MPC clusters in the angular domain, where the clustering of the MPCs is performed based on the cosine-distance between the dominant MPCs.

For a given transmitter (TX) and receiver (RX) pair, the EMR is a single scalar value that characterizes the robustness of the communication link against blockages, as it captures the number of unique communication directions that can be utilized. It is also possible to characterize the blockage robustness for the whole environment by evaluating the spatial distribution of the EMR metric considering various different TX/RX locations. Using our proposed metric, one can assess the scattering richness of different environments to achieve a particular service quality. We evaluate the proposed metric using our $28 \mathrm{GHz}$ channel measurements in a library environment for Line-of-Sight (LOS) and NLOS scenarios, and compare it with some other commonly used propagation metrics. We argue that EMR is especially informative at higher frequencies, e.g., mmWave and terahertz (THz), where the propagation attenuation is high, and directional Non-Light-of-Sight (NLOS) communication is critical for the success of the network.
\end{abstract}

Keywords - $28 \mathrm{GHz}, 5 \mathrm{G}, 6 \mathrm{G}$, angular spread, blockage, delay spread, millimeter-wave (mmWave), multipath components (MPCs), multipath richness

\section{INTRODUCTION}

High-frequency bands, such as millimeterwave (mmWave) and terahertz (THz), have attracted increasing attention as a solution to the continuously growing data rate demand. Due to very large amounts of available spectrum at these higher frequencies, they have received major attention for $5 \mathrm{G}$, and recently $6 \mathrm{G}$, standardization efforts. For efficient planning of wireless networks, a thorough understanding of the propagation channel characteristics in the respective deployment band is critical. Even though comprehensive knowledge of the sub- $6 \mathrm{GHz}$ bands have been acquired already through extensive channel measurements and modeling, there is still much to investigate about mmWave bands for successful deployment and operation of the wireless networks using these bands.

Due to their high frequency, mmWave signals are more sensitive to blockages and attenuate much faster than the sub- $6 \mathrm{GHz}$ signals $[1,2]$. For the same reason, freespace path loss of a typical mmWave link is more than an order-of-magnitude larger than that of a traditional sub-6 GHz link [3]. These factors restrict the number of dominant paths (over which communication can be reasonably achieved within Quality-of-Service (QoS) constraints) at mmWave frequencies to only a few [4], i.e., Line-of-Sight (LOS) path, if available, and a few additional paths through strong reflections from obstacles. The number of dominant paths may further decrease, or there may be none at all, if one or more paths are blocked due to mobile obstacles, as illustrated in Fig. 1. However, it is still possible to establish multi-gigabit links between the user and the serving Base Station (BS)/Access Point (AP) provided that the user location has the minimum necessary Received Signal Strength (RSS) over at least one path. The most popular solution for compensating the additional loss at high frequencies is to use phased array antennas. Phased array antennas can electrically create and steer beams in different directions so that alternate paths (with more gain due to beamforming) can be utilized in case the most preferable path(s) is (are) blocked [3].

Given the foregoing background, the number of backup paths (i.e., useful paths that are dominant over any weak scattered components and which can be utilized when stronger paths are blocked) will increase the chance of establishing and maintaining communication between the users and the BSs/APs. Accurate knowledge of these paths under varying channel conditions is important and 


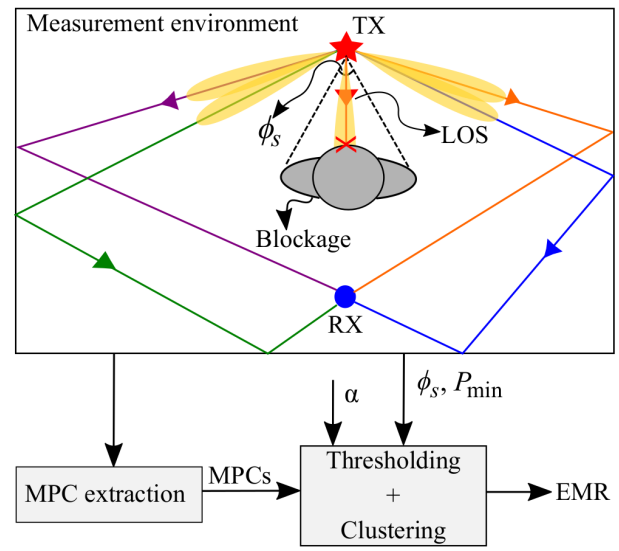

Fig. 1 - An example illustration of the blockage problem and calculation of the EMR metric. There are five paths in total that are above a power level $P_{\min }$ at the RX. The LOS path is blocked and hence the communication should be maintained over the remaining eligible paths. Even though there appears to be four more alternate paths through the reflections from the walls, the two paths on each side can be obscured simultaneously by the same blockage, meaning there are fewer effective backup paths than it appears. The beamwidth is assumed to be smaller than the blockage width $\phi_{s}$. The parameter $\alpha$ defines the relative importance of the weaker paths with respect to the strongest path.

can help to evaluate the suitability of an environment for a particular deployment band or to determine the locations of BSs/APs in a given environment to maximize a performance metric (e.g., coverage or throughput). To address this need, this paper defines a new metric, called Effective Multipath Richness (EMR).

Fig. 1 illustrates the problem and provides an overview of the EMR metric calculation. The EMR metric assesses the value of the multipaths in a measured channel by taking into account the RSS over each path and the overall spatial diversity of the paths. To achieve this, first, paths with power above a certain threshold $\left(P_{\min }\right)$ at the Receiver (RX) are identified and then clustered based on an angular distance metric. The clustering is performed in an iterative manner so that the resulting clusters satisfy a spatial diversity constraint defined by a beam separation threshold $\left(\phi_{s}\right)$. Finally, the relative value of each cluster is determined as a function of the cluster power and a decay coefficient $\alpha$ which determines the relative importance of the weaker paths. The value sum is output as the EMR for a particular Transmitter (TX)/RX location pair.

To represent the overall scattering behavior of an environment, the probability distribution function of the EMR can be used, based on measurements performed at different TX/RX locations in that environment. In essence, the EMR estimates the number of useful paths for communication, considering the environment-specific parameters (e.g., blockage size or probability). It also characterizes the gains that can be obtained via beamforming in case the dominant paths get blocked. It will complement the other well-known metrics, such as the Root-MeanSquare (RMS) Delay Spread (DS) or Angular Spread (AS), to characterize the channel, and accordingly, to plan the deployment and operation of a mmWave network.
The rest of the paper is organized as follows. Section 2 reviews a few well-known metrics that can be used to assess wireless propagation characteristics of an environment and motivates the need for the proposed EMR metric. Section 3 introduces the steps to calculate EMR. Section 4 presents the numerical results on the EMR metric using our data from a $28 \mathrm{GHz}$ channel measurement campaign in an indoor library environment, and Section 5 provides concluding remarks.

\section{CHARACTERIZING THE CHANNEL BEHAVIOR}

Information provided by the Power Delay Profile (PDP) may be mostly sufficient for characterizing the channel at sub- $6 \mathrm{GHz}$ frequencies. However, since the Angle of Arrival/Departure (AoA/AoD) information of the Multipath Components (MPCs) are of critical importance at high frequencies (i.e., due to beamforming), Power Angular-Delay Profiles (PADPs) of the channel should be extracted at these frequency bands. The PADP can be expressed as [5]

$$
\begin{aligned}
P A D P\left(\tau, \theta \mathbf{0}^{\mathrm{AoD}}, \theta \mathbf{0}^{\mathrm{AoA}}\right)= & \sum_{n=1}^{N} \alpha_{n} \delta\left(\theta \mathbf{0}^{\mathrm{AoD}}-\theta \mathbf{0}_{n}^{\mathrm{AoD}}\right) \\
& \times \delta\left(\theta \mathbf{0}^{\mathrm{AoA}}-\theta \mathbf{0}_{n}^{\mathrm{AoA}}\right) \delta\left(\tau-\tau_{n}\right),
\end{aligned}
$$

where $N$ is the number of MPCs, $\alpha_{n}$ is the path gain, $\theta \mathbf{0}_{n}^{\mathrm{AoD}}=\left[\theta_{n}^{\mathrm{AoD}, \mathrm{Az}} \theta_{n}^{\mathrm{AoD}, \mathrm{El} l}\right]^{\top}$ and $\theta \mathbf{0}_{n}^{\mathrm{AoA}}=\left[\theta_{n}^{\mathrm{AoA}, \mathrm{Az}} \theta_{n}^{\mathrm{AoA}, \mathrm{El}}\right]^{\top}$ are the two-dimensional AoD and AoA of the $n$-th MPC in the azimuth and elevation planes, respectively, and $\tau_{n}$ is the delay of the $n$-th MPC. These parameters can be obtained from channel measurements using appropriate channel sounders or estimated from ray tracing simulations along with accompanying MPC extraction algorithms (e.g., peak search algorithm [5], or superresolution techniques $[6,7])$. The relationship between the received power over each path and the corresponding path gain can be written using the link budget as

$$
P_{n}=P_{\mathrm{TX}}+G_{\mathrm{TX}}+G_{\mathrm{RX}}+\alpha_{n}[\mathrm{~dB}], \quad \forall n \in[N],
$$

where $G_{\mathrm{TX}}$ and $G_{\mathrm{RX}}$ are the TX and RX antenna gains, and the TX/RX antennas are assumed to be aligned with the $n$-th MPC's AoD/AoA directions. We note that $\alpha_{n}$ in (1) is the linear gain, whereas, in (2), it is in $\mathrm{dB}$.

Besides PADP, it is also common in the literature to represent different characteristics of the channel concisely using various metrics, such as RMS-DS and RMS-AS. Easyto-interpret metrics can highlight certain aspects of the channel and help in optimizing performance while planning the network. Next, we will briefly review some of these metrics. We will also provide numerical results based on a few representative channel measurements which will motivate the need for our proposed metric. In the rest of the paper, we will assume that the MPCs have already been extracted from channel measurements or ray tracing simulations as discussed above and use the parameters to compute the metrics. 


\subsection{Existing metrics for channel characterization}

RMS-DS is a measure of temporal dispersion of the power. It is calculated using the power and delay parameters of the extracted MPCs as follows [8]:

$$
\tau_{\mathrm{rms}}=\sqrt{\frac{\sum_{n=1}^{N} P_{n}\left(\tau_{n}-\tau_{\mathrm{avg}}\right)^{2}}{\sum_{n=1}^{N} P_{n}}},
$$

where $P_{n}$ is the power of the $n$-th MPC, and $\tau_{\text {avg }}$ is the mean delay given by

$$
\tau_{\text {avg }}=\frac{\sum_{n=1}^{N} P_{n} \tau_{n}}{\sum_{n=1}^{N} P_{n}} .
$$

RMS-AS indicates the dispersion of power in the spatial domain. It can be calculated similarly to the RMS-DS as follows [9]:

$$
\sigma_{\mathrm{rms}}=\sqrt{\frac{\int_{-\pi}^{\pi} P(\omega)\left(\omega-\omega_{\mathrm{avg}}\right)^{2} d \omega}{\int_{-\pi}^{\pi} P(\omega) d \omega}},
$$

where $\omega$ is the AoA either in the azimuth or the elevation plane, and $P(\omega)$ is the sum of linear power of the MPCs whose AoA is $\omega$ in the azimuth or the elevation plane. The term $\omega_{\text {avg }}$ is the average AoA and given by

$$
\omega_{\mathrm{avg}}=\frac{\int_{-\pi}^{\pi} P(\omega) \omega d \omega}{\int_{-\pi}^{\pi} P(\omega) d \omega}
$$

Another relevant metric to understand the scattering characteristics of the environments is the Angular Spread Coverage (ASC) introduced in [10]. This metric is an extension of the AS metric that was originally proposed in [11] and quantifies the spatial diversity of the multipaths by

$$
\mathrm{ASC}=\frac{P_{\max }+\sqrt{\left|F_{0}\right|^{2}-\left|F_{1}\right|^{2}}}{P_{\max }},
$$

where $P_{\max }$ is the power of the MPC with maximum power, and

$$
F_{q}=\int_{-\pi}^{\pi} P(\omega) \exp (\mathrm{j} q w) d \omega
$$

is the $q$-th complex Fourier coefficient of the power arriving at the azimuth angle $\omega$. The original version of the metric does not have the $P_{\max }$ terms in the numerator and the denominator in (7) and hence returns zero if the available paths have the same AoA azimuth angle. However, the ASC metric can capture the event that the client is in coverage if there is at least one available path.

Finally, we consider a slightly modified version of the RMS-AS, which we refer to as the non-normalized RMSAS (nRMS-AS). As its name signifies, the nRMS-AS is calculated as in (5) but without normalization, i.e., the integral
Table 1 - Parameters of the MPCs shown in Fig. 2(a).

\begin{tabular}{lcccccc}
\hline $\begin{array}{l}\text { MPC } \\
\#(\mathrm{i})\end{array}$ & $\begin{array}{c}\text { AoD-Az } \\
\left({ }^{\circ}\right)\end{array}$ & $\begin{array}{c}\text { AoD-El } \\
\left(^{\circ}\right)\end{array}$ & $\begin{array}{c}\text { AoA-Az } \\
\left(^{\circ}\right)\end{array}$ & $\begin{array}{c}\text { AoA-El } \\
\left({ }^{\circ}\right)\end{array}$ & $\begin{array}{c}\text { Power } \\
(\mathrm{dBm})\end{array}$ & $\begin{array}{c}\text { Delay } \\
(\mathrm{ns})\end{array}$ \\
\hline 1 & -90 & 0 & 90 & 0 & -42.00 & 6.51 \\
2 & -150 & 0 & 150 & 0 & -53.02 & 13.67 \\
3 & -150 & 0 & 150 & -20 & -57.40 & 11.72 \\
4 & -30 & 0 & 30 & 0 & -57.67 & 19.53 \\
5 & -90 & -20 & 90 & -20 & -58.74 & 7.16 \\
6 & -110 & 0 & -110 & 0 & -60.46 & 24.74 \\
\hline
\end{tabular}

in the denominator of (5) is set to unit power. The intuition here is that, due to the high attenuation in mmWave frequencies, actual power of the MPCs is a critical feature, and normalizing weighted powers by the total power may remove the multipath richness-related aspects of the channel we are looking for. Given that, the nRMS-AS can be thought of as the RMS value of the absolute power in the angular domain. A similar procedure can be followed to obtain the non-normalized RMS-DS, but we omit this metric in this study for brevity.

\subsection{Evaluation of the metrics}

This section compares the above metrics evaluated for three scenarios, where the number of extracted MPCs and their spatial distribution vary between the scenarios. Fig. 2(a) shows the first six strongest MPCs (both at the TX and the RX sides) extracted from a sample channel measurement at $28 \mathrm{GHz}$. The measurement was conducted in a typical office room of size $\approx 5 \mathrm{~m} \times 8 \mathrm{~m}$ and equipped with furniture and computers. To avoid any noise in the measurements, the measurement setup was operated through remote control. Even though we performed the measurements in indoor environments, we inspected the spectrum to make sure that there is no other signal source around operating at $28 \mathrm{GHz}$. The highest power is received through the LOS path and denoted by the red arrow. Other paths are through first-order reflections from either the surrounding walls and furniture or the ground, and their power levels are determined by the length of the paths and the material type of the reflectors. It is worthwhile to note that there is no second or higher-order reflections in the list due to severe attenuation at $28 \mathrm{GHz}$. Parameters of the MPCs are provided in Table 1. This scenario (Scenario 1) is modified by assuming that the Scatterer \#1 is not a good reflector, and thus paths 2 and 3 are not available. It is also assumed that the remaining paths have the same parameters as in Scenario 1 . This scenario is referred to as Scenario 2 and illustrated in Fig. 2(b). Lastly, Scenario 1 is also modified such that there is a blockage between the TX and the RX, which obscures the LOS path, the ground-reflected path, and the path reflected from Scatterer \#3. Therefore, there are only three paths (with the same parameters) as shown in Fig. 2(c). This scenario is referred to as Scenario 3. 


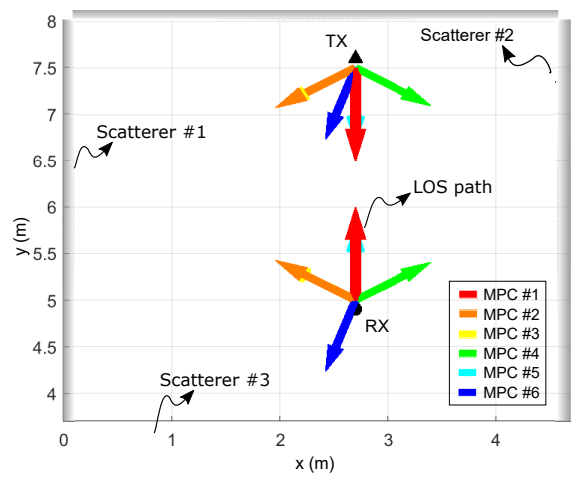

(a) Scenario 1

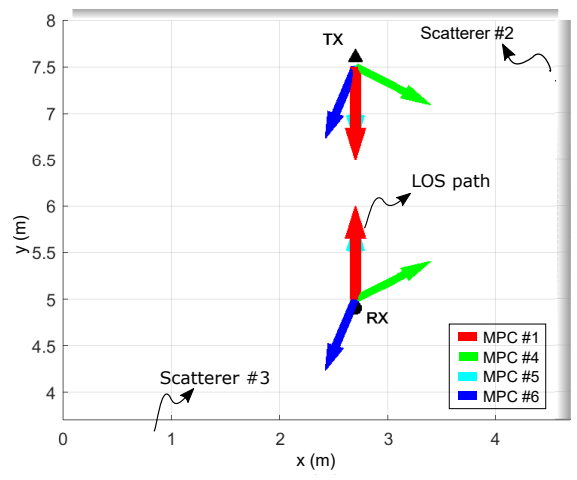

(b) Scenario 2

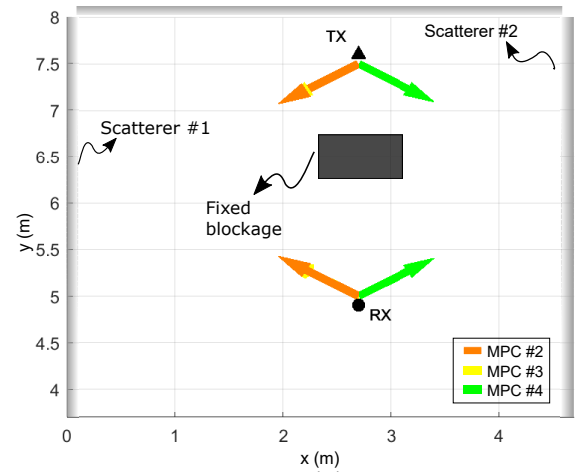

(c) Scenario 3

Fig. 2 - MPCs in the azimuth plane for three different scenarios. Elevation plane is omitted. MPCs are ordered and colored according to their power $P_{n}$. Warmer colors represent higher power. The MPCs with the same color in different scenarios have the same parameters. The MPC set and hence the spatial/temporal diversity of the paths differ in each scenario; however, RMS-DS, RMS-AS, and ASC fail to reflect these differences, and none of them provides information about the number of backup paths. On the other hand, the EMR can differentiate between the scenarios in terms of their true multipath richness and identifies Scenario 1 as the best environment wih an EMR of 2.42, whereas the other two scenarios have smaller (and comparable) EMRs.

Table 2 - Comparison of the EMR with the other metrics for the three scenarios in Fig. 2.

\begin{tabular}{lccccc}
\hline & $\begin{array}{c}\text { RMS-DS } \\
(\mathrm{ns})\end{array}$ & $\begin{array}{c}\text { RMS-AS } \\
\left({ }^{\circ}\right)\end{array}$ & $\begin{array}{c}\text { nRMS-AS } \\
\left({ }^{\circ}\right)\end{array}$ & ASC & EMR \\
\hline Sce\#1 & 3.29 & 26.28 & 0.23 & 1.46 & 2.42 \\
Sce\#2 & 2.91 & 20.87 & 0.17 & 1.29 & 1.64 \\
Sce\#3 & 2.67 & 48.05 & 0.14 & 2.18 & 1.81 \\
\hline
\end{tabular}

The value of the metrics for the above scenarios are given in Table 2. RMS-AS, nRMS-AS, and ASC metrics are calculated based on the azimuth angles. Since it is a small indoor environment and the measurement was taken at a high frequency (i.e., $28 \mathrm{GHz}$ ), RMS-DS values are also small. Moreover, RMS-DS values are close to each other for all three cases. The number of available MPCs and hence the overall spatial distribution vary depending on the environment (e.g., blockage locations); however, RMS-DS does not change significantly, as it represents the delay spread around the mean delay.

In a similar way to the RMS-DS, although the first two strongest MPCs following the LOS path are missing in Scenario 2, there is no much difference between the RMS-AS values of Scenario 1 and Scenario 2. Due to the blockage in Scenario 3, three paths, including the LOS path, are obscured. Therefore, power levels of the remaining MPCs become more comparable (see Table 1). Besides, when the three MPCs are removed, the remaining MPCs become more spread in the angular domain. As a result, a notable increase is observed in the RMS-AS in the last scenario. Similar to the RMS-AS, the ASC metric returns the maximum value for the last scenario. Although the multipath richness is the highest in the first scenario, the first two scenarios have close ASC values. On the other hand, the nRMS-AS is observed to better reveal the channel characteristics we seek out. That is, the nRMS-AS is the highest for Scenario 1, where the spatial diversity is the largest among the other scenarios. Also, the nRMS-AS values are very close to each other for Scenario 2 and Scenario 3, indicating that the two channels are similar to each other in terms of the spatial diversity of the MPCs. However, since the nRMS-AS is a non-normalized metric, its value depends on the absolute power of the MPCs, making it inappropriate to be used to compare the channels where there are large differences in the MPC power levels.

Despite bringing valuable insights into power dispersion in spatial/temporal domains, none of the above metrics provide information about the number of alternate paths and how valuable the paths are when a particular environment is considered. As discussed above, the metrics may return similar values for different sets of MPCs. In addition, relying only on these metrics, it is not straightforward to estimate the channel behavior against different sizes of blockages and the likelihood of a user being in outage. Therefore, we propose the EMR metric, which takes into account the power level of the backup paths and their angular separation, and assesses the relative value of each individual path with respect to the strongest path. The EMR values for the three scenarios are provided in Table 2 and will be interpreted in Section 4.

We note that the AS needs to be calculated separately for azimuth and elevation planes, whereas the EMR metric is calculated based on the three-dimensional angular distance between the paths. However, since the environmental parameters, such as user density or the blockage size/distribution, may differ in different parts of the environments, the EMR should be computed for both the AoA and the AoD for a more complete characterization of the channel. We explain the procedure to calculate the EMR in the following section for only the AoD (i.e., at the TX side). The procedure for the AoA is the same except that, while clustering the MPCs, angular distance between the paths are calculated based on the AoA instead of AoD. 


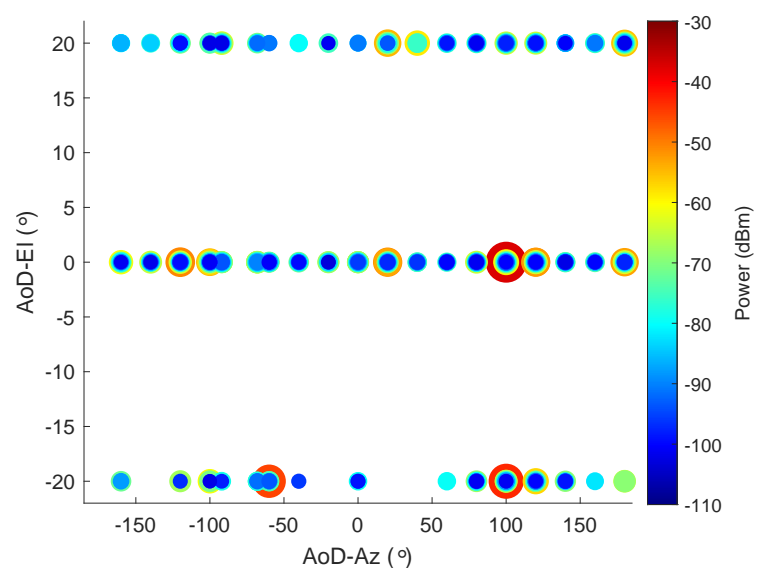

(a)

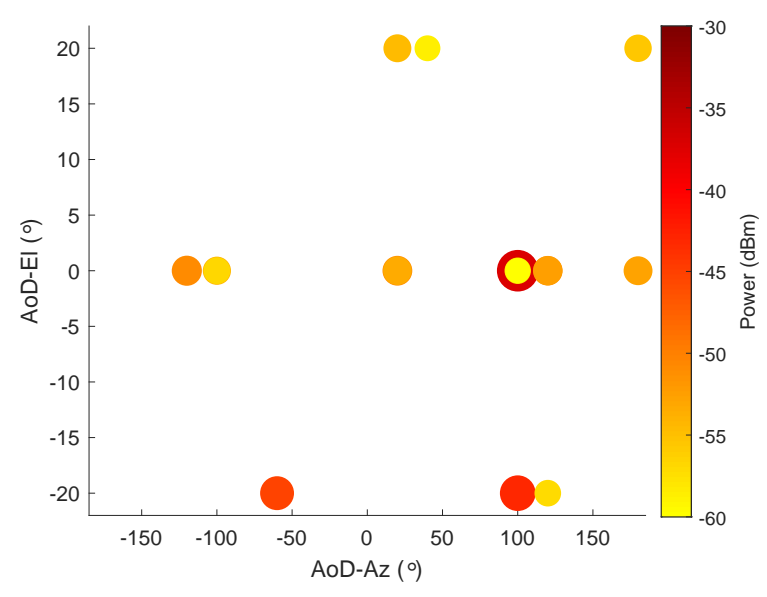

(b)

Fig. 3 - MPCs in the AoD-power space: (a) All MPCs and (b) MPCs after thresholding with $P_{\min }=-60 \mathrm{dBm}$. Points with multiple colors indicate multiple MPCs at the same point.

\section{EFFECTIVE MULTIPATH RICHNESS (EMR)}

To calculate the EMR, two user-defined inputs are required: a minimum power level (at the RX) for the MPCs, and a beam separation threshold. The first input is used to select the MPCs through which the communication can be maintained, whereas the second one is used in clustering the MPCs to estimate the number of backup paths that are well-spread over the angular space. Following the power thresholding and clustering processes, the EMR is expressed as the sum of the weighted number of clusters, and the effective richness of the channel in terms of useful paths is revealed. The procedure to find the EMR is explained in detail next. The outcomes of the intermediate steps are demonstrated using a sample measurement (performed in a library environment for TX1-RX1 pair shown in Fig. 5(a)). We acknowledge that, in this study, we are only interested in the dominant MPCs and their overall spatial diversity. That is, we are not concerned with whether the MPCs reach the receiver through diffraction, diffuse or specular scattering, or after penetrating through obstacles.

\subsection{Thresholding with the minimum desired power level}

As beamforming will be used at higher frequencies, it would be misleading to include the low-power paths in the calculation of the EMR because these paths will not be of any (or only be a little) help to the communication performance. Therefore, after the extraction of the MPCs, the first step is to identify the MPCs that are above a minimum received power level. This power level should be determined based on the application-specific communication needs, operating frequency, and the available environmental parameters, such as the blockage size/probability statistics. For example, in [12], where a learning-assisted beam search scheme is proposed for indoor mmWave networks at $60 \mathrm{GHz}$, the minimum required RSS is defined as $-60 \mathrm{dBm}$; otherwise the User Equipment (UE) is triggered to find a stronger link. On the other hand, in [13], the RSS threshold is considered to be $-84 \mathrm{dBm}$ for a reliable communication at $60 \mathrm{GHz}$ between the vehicles and the Road Side Units (RSUs) in an urban environment from Manhattan, NY. Similar to $[12,13]$, we consider a threshold in this work to discard paths in the angular domain that are weaker than that threshold. In Section 4, we will investigate how the EMR changes with different thresholds.

Let $\mathbf{X}=\left\{\chi_{1}, \chi_{2}, \ldots, \chi_{N}\right\}$ be the set of all MPCs with $N$ being the total number of MPCs. Each MPC in $\mathbf{X}$ can be characterized by six parameters as follows:

$$
\chi_{n}=\left\{\theta_{n}^{\mathrm{AoD}, \mathrm{Az}}, \theta_{n}^{\mathrm{AoD}, \mathrm{El}}, \theta_{n}^{\mathrm{AoA}, \mathrm{Az}}, \theta_{n}^{\mathrm{AoA}, \mathrm{El}}, \tau_{n}, P_{n}\right\},
$$

where $n=1, \ldots, N$. Then, the subset of MPCs whose power are above a predetermined level $P_{\min }$ can be defined as

$$
\overline{\mathbf{X}}=\left\{\chi_{n} \mid P_{n}>P_{\min }, n=1, \ldots, N\right\}
$$

Fig. 3(a) shows all the MPCs (extracted from the measurements shown in Fig. 5(a) at TX1-RX1 pair) in the AoD-power space. We note that the number of extracted MPCs depends on several factors, such as multipath delay resolution of the channel sounder or the MPC extraction algorithm. Based on the choice of these factors, one can end up with a much smaller number of MPCs than shown in Fig. 3(a). Considering the requirements, such as minimum average date rate per user, let us assume the minimum power level $P_{\min }$ for an MPC to be eligible as a backup path (after a blockage, in a different beam direction) to be $-60 \mathrm{dBm}$. Then, the number of MPCs reduces to only 17, and the resulting MPCs are shown in Fig. 3(b). The parameters of these MPCs are provided in Table 3. As it is clear from Fig. 3(b), three-dimensional AoDs for some of the MPCs are very close to each other, which may result in multiple MPCs being blocked in the presence of an obstacle (of certain size) that is at a certain distance to the TX. As a result of this observation, the likelihood of 
Table 3 - MPCs with power level above $P \min =-60 \mathrm{dBm}$ and cosine distance-based clustering results for $\phi_{s}=20^{\circ}$.

\begin{tabular}{lccccccc}
\hline MPC & $\begin{array}{c}\text { AoD-Az } \\
\#(\mathrm{i})\end{array}$ & $\begin{array}{c}\text { AoD-El } \\
\left.{ }^{\circ}\right)\end{array}$ & $\begin{array}{c}\text { AoA-Az } \\
\left.{ }^{\circ}\right)\end{array}$ & $\begin{array}{c}\text { AoA-El } \\
\left.{ }^{\circ}\right)\end{array}$ & $\begin{array}{c}\text { Power } \\
\left({ }^{\circ}\right)\end{array}$ & $\begin{array}{c}\text { Delay } \\
(\mathrm{dBm})\end{array}$ & $\begin{array}{c}\text { Cluster } \\
(\mathrm{ns})\end{array}$ \\
\hline 1 & 100 & 0 & -80 & 0 & -37.33 & 33.85 & 1 \\
2 & 100 & -20 & -80 & 0 & -43.25 & 33.20 & 1 \\
3 & -60 & -20 & -80 & -20 & -45.35 & 42.32 & 2 \\
4 & -120 & 0 & -80 & 0 & -50.75 & 57.94 & 4 \\
5 & 20 & 0 & -20 & 0 & -51.27 & 88.54 & 3 \\
6 & 120 & 0 & -100 & 0 & -51.60 & 37.11 & 1 \\
7 & 120 & 0 & -120 & 0 & -52.35 & 39.06 & 1 \\
8 & 180 & 0 & -140 & 0 & -52.83 & 115.88 & 5 \\
9 & 20 & 0 & -140 & 0 & -53.42 & 102.21 & 3 \\
10 & -100 & 0 & -80 & 0 & -54.08 & 54.68 & 4 \\
11 & 20 & 20 & -20 & 20 & -54.52 & 94.40 & 3 \\
12 & 180 & 20 & -20 & 20 & -55.47 & 107.42 & 5 \\
13 & -100 & 0 & -80 & 0 & -56.60 & 55.99 & 4 \\
14 & 120 & -20 & -100 & 0 & -57.07 & 36.46 & 1 \\
15 & 100 & 0 & -120 & 0 & -57.50 & 38.41 & 1 \\
16 & 40 & 20 & -20 & 20 & -58.69 & 93.74 & 3 \\
17 & 100 & 0 & 120 & -20 & -59.93 & 40.36 & 1 \\
\hline
\end{tabular}

finding a link between the TX and the RX will be higher when the MPCs spread out over the AoD azimuth and AoD elevation space compared to when the MPCs exhibit clusters. Therefore, for a reliable assessment of the multipath richness of a channel, one should also take into consideration the spatial diversity of the MPCs.

\subsection{Cosine distance-based clustering}

Having identified the paths over which a link can be established, the next step is to find the number of effective alternate paths (i.e., beam directions that are separated by at least a user-defined angular distance from any other). Here we assume that the beamwidth is smaller compared to the blockage angle $\phi_{s}$, as shown in Fig. 1. To achieve higher gains, multiple antenna elements with narrow beams are used at mmWave frequencies. This also limits the number of beam directions. For example, it is shown in [14] that the number of beam directions at the mmWave BS side (with a $8 \times 8$ array and beamwidth $13^{\circ}$ ) is only 10 in azimuth when the BS scans a total of 120 degrees.

Unlike the approaches that aim to parameterize the channel impulse response by clustering the MPCs based on AoA, AoD, and delay information (e.g., $[15,16])$, we cluster the MPCs only using their angular parameters. This way, it is possible to find how many alternate paths are available if some of the paths are blocked. Omitting the parameters other than the angles in the interested domain (in this case, the AoD), each MPC in $\overline{\mathbf{X}}$ in (10) can be represented by a vector in three-dimensional space as

$$
\chi_{l}=\left\{r_{l}, \theta_{l}^{\mathrm{AoD}, \mathrm{Az}}, \theta_{l}^{\mathrm{AoD}, \mathrm{El}}\right\},
$$

for $l=1, \ldots, L$, where $L$ is the number of MPCs in $\overline{\mathbf{X}}$, and $r_{l}$ is the magnitude of the vector $\chi_{l}$. While $r_{l}$ may be defined as the individual powers of the MPCs, since here only the angles are of interest, $r_{l}$ can be simply set to 1 , so $\chi_{l}$ becomes a unit vector with the given elevation and azimuth angles.

For clustering the MPCs in (11), an iterative cosine distance-based k-means clustering algorithm is used. At each iteration $\overline{\mathbf{X}}$ is grouped into a number of clusters that is equal to the current iteration count, and this process is repeated until the desired spatial distance between the cluster centroids is achieved. The cosine distance between any two vectors $a$ and $b$ is defined as follows:

$$
d(a, b)=1-\cos (\phi),
$$

where $\phi$ is the angle between the vectors in threedimensional space. If $a$ and $b$ point in the same direction $\left(\phi=0^{\circ}\right)$, then $d(a, b)=0$, or if they point in opposite directions $\left(\phi=180^{\circ}\right)$, then the distance attains its maximum, and $d(a, b)=2$.

Let $\boldsymbol{\Gamma}_{\boldsymbol{\Gamma}}=\left\{c_{k, 1}, \ldots, c_{k, L}\right\}$ be the set of cluster centroids at $k$-th $(k \leq L)$ iteration, where $c_{k, l}$ is a $1 \times 3$ vector that represents the centroid of $\chi_{l} \in \overline{\mathbf{X}}$. It should be noted that, although each MPC will be matched to a centroid, there will be $k$ unique clusters and hence centroids. Each centroid is the coordinate-wise mean of the points in a cluster, after normalizing those points to unit Euclidean length. Once the centroids are found for the current number of clusters, the angular distance between any MPC and the centroid of the cluster to which that MPC belongs is calculated. If all the MPCs are at an angular distance from their centroids of less than a beam separation threshold $\phi_{s}$, i.e.,

$$
\phi_{k, l}=\angle c_{k, l} \chi_{l}<\phi_{s}, \forall l \in(1, \ldots, L),
$$

then the iterations are terminated, and the current iteration count $k$ is returned as the number of clusters.

The parameter $\phi_{s}$ is a design parameter, and it represents the size or the angular width of the blockages that are likely to obscure the possible links between the TX and the RX in a given environment (see Fig. 1). When $\phi_{s}$ is higher, it gets more likely that the paths close to each other will be blocked. As a result, there will be fewer paths over which the signals can be transmitted. We point out that if the angular resolution of the TX/RX antennas is much lower than $\phi_{s}$, then the clustering process can be skipped. In such a case, each resolved path can be treated as a cluster, and their powers can be directly plugged into (14) to calculate the EMR.

It should be recalled that initializing the centroids at different locations may lead the k-means algorithm to return different clusters. It is also possible that some initializations may result in local optima. Both scenarios introduce a bias in the EMR values. Therefore, at each iteration, we randomly initialize $k$ centroids, run the k-means algorithm, and compute the cost function. This process is repeated $K$ times, and the clustering that yields the lowest cost is picked for the current iteration. For the channel measurements used in this study, we observed that it is sufficient to set $K=20$ for the clusters to converge; 


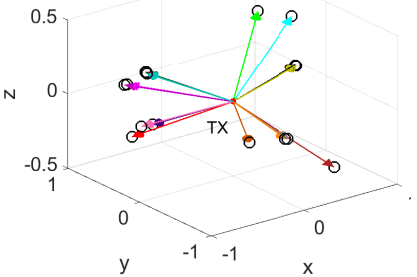

(a)

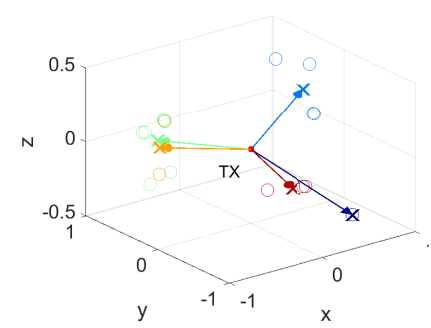

(c)

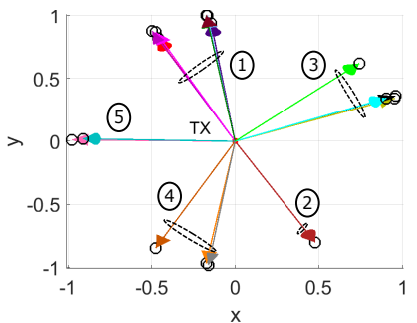

(b)

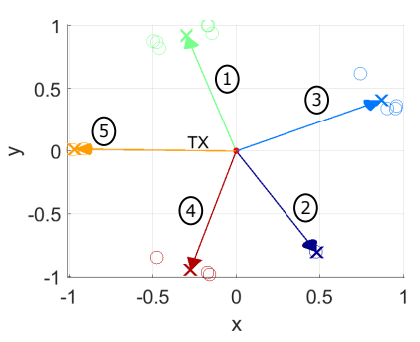

(d)
Fig. 4 - MPCs (in unit vector representation) after thresholding with $P_{\min }=-60 \mathrm{dBm}(\mathrm{a})$ in 3D and (b) in 2D. Representation of the MPCs by centroids after our clustering algorithm with $\phi_{s}=20^{\circ}$ (c) in 3D and (d) in 2D. The centroids after clustering in (d) are more evenly spread out and hence they better capture the number of beam directions compared to (b) for the given $\phi_{s}$.

however, larger $K$ values may be required if there are too many eligible paths after the thresholding step.

We applied our clustering algorithm on the MPCs listed in Table 3. The stoppage criterion in (13) is satisfied after $k=5$ iterations, when $\phi_{s}$ (characterizing the blockage angle) is set to $20^{\circ}$, and the cluster IDs of the MPCs are given in the last column of the table. Thus, when the resilience of the channel to blockages and hence the spatial diversity of the useful MPCs are of concern, for the given $\phi_{s}$ it may be presumed that there are only four alternate beam directions outside of the dominant beam direction. The MPCs in unit vector representation after thresholding and clustering steps are shown in 3D view in Fig. 4(a) and Fig. 4(c), respectively, and in 2D view along with the cluster IDs in Fig. 4(b) and Fig. 4(d). In Fig. 4(a) and Fig. 4(b), MPCs are indicated with a circle and the corresponding unit vectors, whereas the cross marks in Fig. 4(c) and Fig. 4(d) denote the centroids of the clusters. By comparing Fig. 4(b) and Fig. 4(d) and treating the cen- troids as new MPCs, it can be seen that the new MPCs are more evenly spread out and hence better summarize the usable number of backup paths for the given $\phi_{s}$.

\subsection{Value of the backup paths and the EMR metric}

The last step in calculating the EMR is to compute the value of the backup paths. We note that, after the clustering process, backup paths refer to the cluster centroids with the difference that the magnitude term $r_{l}$ in (11) is now the linear sum of the powers of the MPCs in the cor-
Table 4 - Value of each cluster and the EMR for different $\alpha$ values $\left(\phi_{s}=\right.$ $20^{\circ}$ and $P \min =-60 \mathrm{dBm}$ ).

\begin{tabular}{lcccc}
\hline $\begin{array}{l}\text { Cluster } \\
\#(\mathrm{i})\end{array}$ & $\begin{array}{c}\text { Cluster power } \\
(\mathrm{dBm})\end{array}$ & $\begin{array}{c}\text { Value } \\
(\alpha=0.1)\end{array}$ & $(\alpha=0.4)$ & $(\alpha=0.7)$ \\
\hline 1 & -36.02 & 1.00 & 1.00 & 1.00 \\
2 & -45.35 & 0.80 & 0.42 & 0.22 \\
3 & -47.72 & 0.76 & 0.33 & 0.15 \\
4 & -48.39 & 0.75 & 0.31 & 0.13 \\
5 & -50.94 & 0.70 & 0.24 & 0.08 \\
\hline \hline & $\rho \mathbf{0}$ & $\mathbf{4 . 0 1}$ & $\mathbf{2 . 3 0}$ & $\mathbf{1 . 5 8}$ \\
\hline
\end{tabular}

responding cluster. The value of each backup path depends on its power level relative to the strongest path in the same measurement. Let $P_{c, m}$ be the power of the $m$ th cluster and $K$ be the total number of clusters. Then, the EMR is calculated by summing the value of the individual paths as follows:

$$
\rho=\sum_{m=1}^{K}\left(\frac{P_{c, m}-P_{\min }}{P_{c, \max }-P_{\min }}\right)^{\alpha},
$$

where $P_{c, \text { max }}$ is the power of the strongest cluster, and $\alpha$ is the decay coefficient that takes values in $[0,1]$. Min-max normalization in (14) ensures that the maximum value of any cluster is limited to 1 . On the other hand, $\alpha$ is a tunable parameter that determines the relative value of the clusters with respect to the cluster with the highest total power. If $\alpha=0$, then the value of each cluster will be the same and equal to 1 . As $\alpha$ is increased towards 1 , the value of the weaker clusters and hence the EMR decreases. It is also important to stress that, in normalizing the cluster powers, $P_{\min }$ is used instead of $P_{c, \min }$. This way, while determining the value of the cluster MPCs, the metric also takes into account how much they are above the minimum required power $P_{\min }$.

Table 4 shows the power and the value of each cluster (i.e., the summand in (14)) or the backup paths, and the EMR for three different $\alpha$ values when $\phi_{s}=20^{\circ}$ and $P_{\min }=$ $-60 \mathrm{dBm}$. The cluster with the highest power, which includes the LOS path for this sample measurement, has a value of 1 independent of the $\alpha$ value. Therefore, if there is at least one path above $P_{\min }$, then the EMR will be greater than or equal to 1 . As $\alpha$ is increased from 0.1 to 0.7 , the relative value of the weaker clusters decreases in accordance with the above explanation, and the EMR representing this particular TX/RX location decreases from 4.01 to 1.58 . So the value of $\alpha$ can be determined based on how much one wants to rely on the weaker paths to establish communication in a given environment, and the decision on the suitability of a TX location or the whole environment can be made by interpreting the EMR metric.

\section{NUMERICAL RESULTS}

In this section, we evaluate the EMR metric using our channel measurements at the Hunt library at NC State University Centennial Campus for the TX-RX locations shown in Fig. 5. 


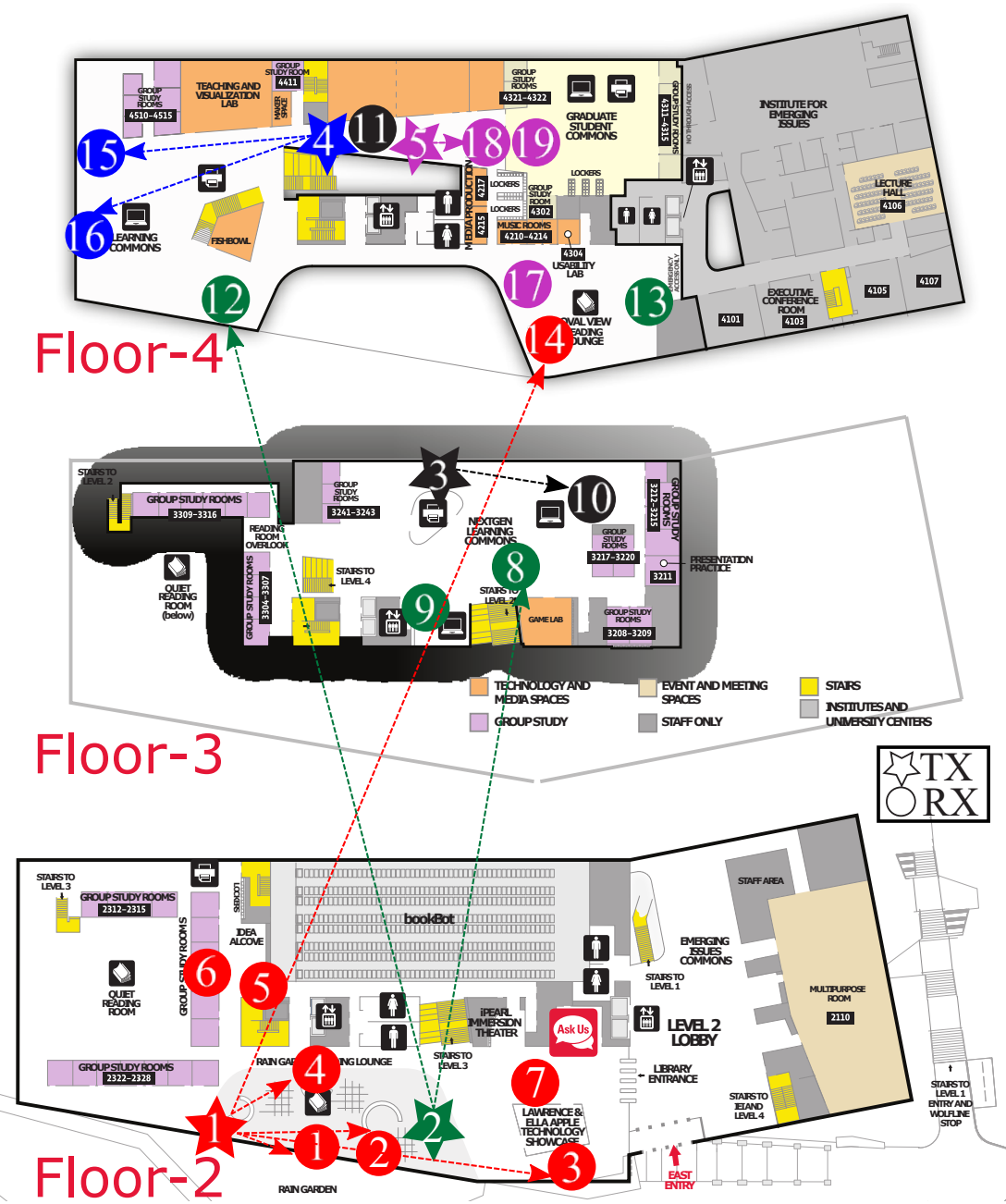

(a)

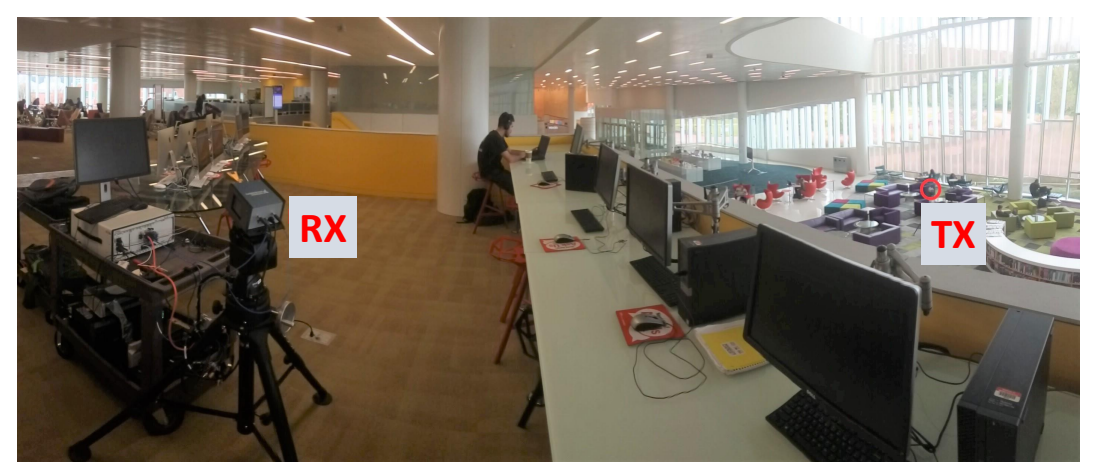

(b)

Fig. 5 - (a) The Hunt library floor plans with TX/RX locations for a total of 19 LOS and NLOS measurements. TXs and RXs that form a pair are indicated with the same color. LOS links are indicated with an arrow pointing from the TX to the corresponding RX. (b) Snapshot of the measurement scenario for TX1-RX3 pair.

We also make a comparison between the EMR and the other metrics discussed in Section 2. The measurement campaign includes 11 LOS and 8 NLOS scenarios for TX-RX separation distances ranging from $10 \mathrm{~m}$ to $50 \mathrm{~m}$. Other details about the measurements and the measurement environment can be found in [17], where we also presented two measurement-based large-scale fading path loss models. For the extraction of the MPCs, we used the peak searching algorithm in [5]. 
Table 5 - Evaluation of the metrics for the library channel measurements.

\begin{tabular}{|l|l|l|l|l|l|l|}
\hline Location & Link State & RMS-DS & RMS-AS & nRMS-AS & ASC & EMR \\
\hline \hline TX1-RX1 & LOS & 32.10 & 30.15 & 0.56 & 1.74 & 2.30 \\
\hline TX1-RX14 & LOS & 29.90 & 81.58 & 0.46 & 5.46 & 2.27 \\
\hline TX1-RX2 & LOS & 38.93 & 48.69 & 0.54 & 1.84 & 2.42 \\
\hline TX1-RX3 & LOS & 34.42 & 63.17 & 0.40 & 2.80 & 1.41 \\
\hline TX1-RX4 & LOS & 36.61 & 49.82 & 0.65 & 1.93 & 2.70 \\
\hline TX2-RX12 & LOS & 37.50 & 92.38 & 0.71 & 4.50 & 1.86 \\
\hline TX2-RX8 & LOS & 29.08 & 154.60 & 1.61 & 3.98 & 3.25 \\
\hline TX3-RX10 & LOS & 44.40 & 53.55 & 0.46 & 3.19 & 1.43 \\
\hline TX4-RX15 & LOS & 27.53 & 38.89 & 0.86 & 2.62 & 2.40 \\
\hline TX4-RX16 & LOS & 32.52 & 72.82 & 0.67 & 9.06 & 1.85 \\
\hline TX5-RX18 & LOS & 29.93 & 88.68 & 1.30 & 3.32 & 2.23 \\
\hline \hline TX1-RX5 & NLOS & 33.91 & 93.95 & 0.36 & 3.55 & 1.30 \\
\hline TX1-RX6 & NLOS & 49.74 & 109.00 & 0.06 & 16.86 & 0.00 \\
\hline TX1-RX7 & NLOS & 41.33 & 54.09 & 0.42 & 3.95 & 1.33 \\
\hline TX2-RX13 & NLOS & 62.21 & 41.30 & 0.09 & 7.23 & 0.00 \\
\hline TX2-RX9 & NLOS & 25.31 & 67.49 & 0.93 & 2.39 & 2.03 \\
\hline TX3-RX11 & NLOS & 59.99 & 71.35 & 0.03 & 7.50 & 0.00 \\
\hline TX5-RX17 & NLOS & 47.03 & 50.82 & 0.13 & 3.211 & 1.00 \\
\hline TX5-RX19 & NLOS & 38.28 & 75.10 & 0.53 & 3.34 & 1.04 \\
\hline
\end{tabular}

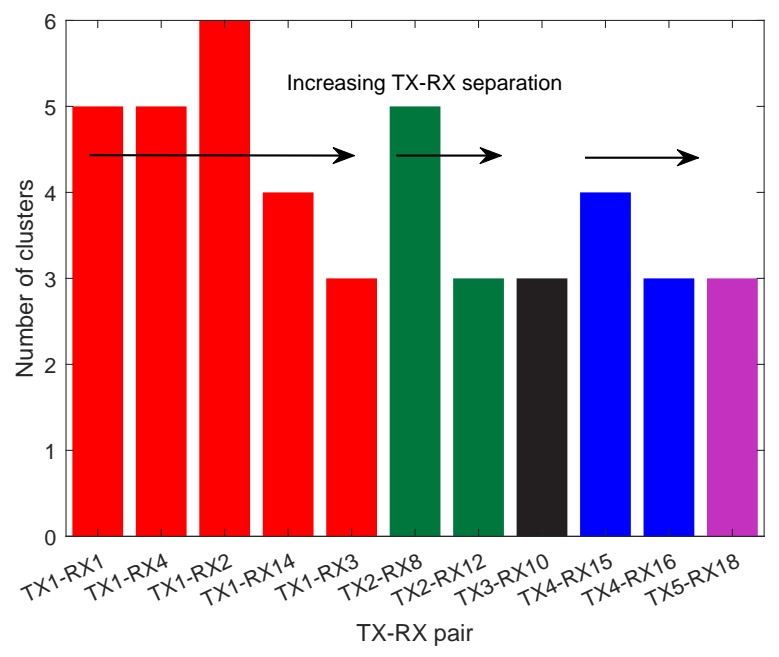

Fig. 6 - Number of clusters for the LOS library measurements using the cosine distance-based clustering in Section $3.2\left(P_{\min }=-60 \mathrm{dBm}, \phi_{s}=\right.$ $20^{\circ}$ ) . The same color code is used for the TX locations as in Fig. 5(a). $\mathrm{RX}$ locations are grouped by the TX location and then ordered by ascending TX-RX separation distance if there are multiple LOS measurements at that TX location. Number of clusters follow a decreasing trend with the increase in TX-RX distance.

\subsection{Number of MPC clusters in angular domain}

We start our analysis by calculating the number of MPC clusters. We focus on only the LOS measurements. Fig. 6 shows the number of clusters for each TX-RX pair calculated using the method in Section 3.2. RXs are grouped based on the TX locations and ordered such that the TX-RX separation increases from left to right in the figure. While identifying the clusters, only the MPCs with a power level of above $-60 \mathrm{dBm}$ were taken into consideration and an angular separation (between each cluster) constraint of $20^{\circ}$ is imposed. As there are other fac- tors along with the TX-RX distance that affect the number of clusters (e.g., reflection properties of the surrounding objects for each TX-RX location), it would be inappropriate to make a generalization about the number of clusters solely based on the TX-RX distance. However, when the RXs are grouped by the TXs as in Fig. 6, it can be easily seen that the number of clusters tends to decrease as TX-RX distance increases. In addition, it can be observed from the figure that, independent of the TX-RX separation distance (up to $50 \mathrm{~m}$ ) and height difference (up to two floors), there are at least three well-separated paths that can be utilized for all the scenarios. Depending on the surrounding furniture, walls, and electrical appliances, the number of alternate paths increases up to six.

Although the above observations are of considerable value, they are not sufficient to reveal the relative importance of the weaker paths and hence the true multipath richness of a channel. For example, it is highly likely to have multiple TX locations that will lead to the same number of clusters for the same RX location, and in such a case, the information about the number of clusters does not provide sufficient insight into which TX location is more favorable than the other. For this reason we use the EMR metric but we also note interpreting the EMR with the number of clusters can provide additional insights on the propagation channel.

\subsection{Comparison of EMR with the other metrics}

Before proceeding with the evaluation of the metrics for the library measurements, we go back to Table 2 where we compare the EMR metric with the existing metrics for the scenarios shown in Fig. 2. When the TX side is considered with parameters $\left\{\alpha, \phi_{s}, P_{\min }\right\}=$ 


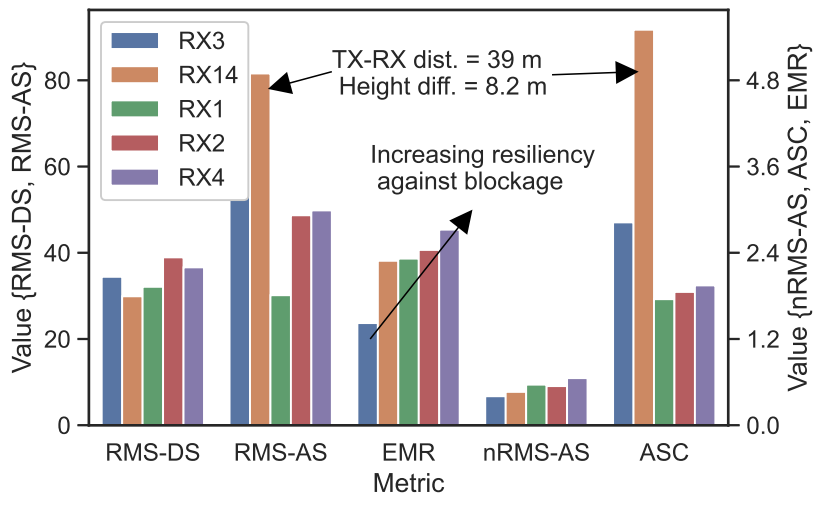

(a)

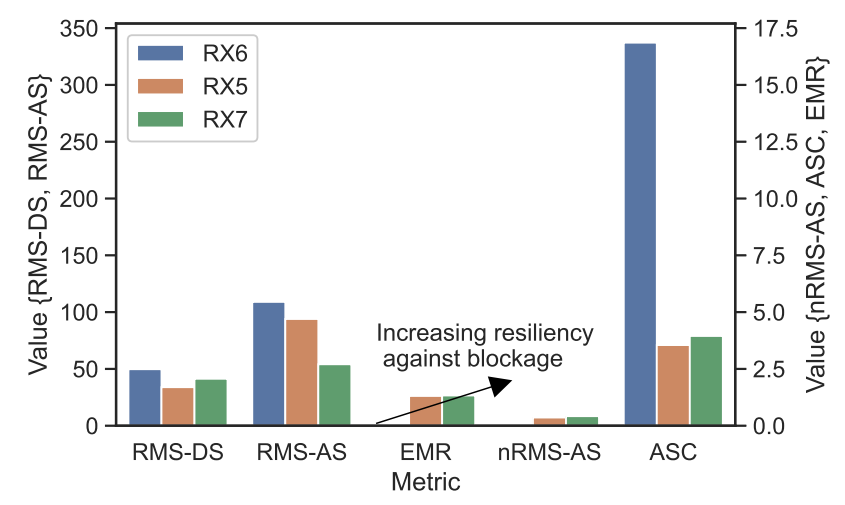

(b)

Fig. 7 - The metrics evaluated based on the scenarios where the TX is located at TX1 with (a) RXs in LOS with the TX1, and (b) RXs in NLOS with the TX1. The EMR values are calculated using the following parameters: $\phi_{s}=20^{\circ}, P_{\min }=-60 \mathrm{dBm}, \alpha=0.4$.

$\left\{0.1,20^{\circ},-60 \mathrm{dBm}\right\}$, the EMR is equal to 2.42 , which means that, in addition to the LOS path, there are at least two well-separated paths with power above the required minimum. This is because, from (14), the value of a backup path can be equal to at most 1 so one can conclude there are at least $\lfloor\rho\rfloor$ backup paths, where $\lfloor\cdot\rfloor$ is the floor function. For example, if the LOS path (MPC \#1) is blocked, then most likely the paths of MPC \#5 and MPC \#6 will be blocked as well; however, the EMR indicates that the communication can be maintained over two alternate paths which are separated by more than $20^{\circ}$ from any other path. Similarly, as it can be seen from Fig. 2(b) and Fig. 2(c), MPCs can be clustered into two in both Scenario 2 and Scenario 3. Therefore, the EMR values are close to 2 in both cases indicating the availability of at least one backup path. The EMR value is slightly larger in Scenario 3 than in Scenario 2 because the total power of the clusters are closer to each other in Scenario 3 than in Scenario 2, increasing the value of the weaker cluster.

The metrics calculated based on the library measurements when the TX is placed at location TX1 with the RXs in LOS and NLOS are presented in Fig. 7(a) and Fig. 7(b), respectively. The left ordinates in the figures represent the values for RMS-DS and RMS-AS, whereas the right or- dinates represent the values for the other metrics. As a reference, the complete list of values are presented in Table 5 along with the link state between each TX-RX pair. Consistently with the results for the sample scenarios in Fig. 2, we observe that the metrics other than the nRMSAS exhibit completely different trends than the EMR. Only nRMS-AS follows the same trend as the EMR both in LOS and NLOS scenarios. However, as already discussed in Section 2.2, it is not possible to extract any information about the number of alternate paths from the nRMS-AS. Comparing the metrics for the LOS and NLOS scenarios, it can be seen that the EMR (as well as the nRMS-AS) is lower for the NLOS cases as expected. On the other hand, all the other metrics return higher values for the NLOS measurements, making it misleading to interpret the results in terms of effective multipath richness of the channel.

Among the LOS measurements in Fig. 7(a), location RX14 returns the highest values for the RMS-AS and ASC metrics, which can be credited to the relatively long TX-RX separation distance and the reflections from the ceiling and the surrounding walls. The multipath component powers become comparable as the TX-RX separation distance increases and there are strong reflections from the ceiling and walls, increasing the spatial diversity of the paths. The same result can be observed for TX2-RX12 and TX2-RX8 (see Table 5), where the TXs and RXs are placed at different floors and there is a ceiling above the RXs. However, when the goal is to capture the effective multipath richness and resilience of the paths to blockages, both the RMS-AS and ASC fail. The trends for the two metrics are almost the opposite of the EMR both for the LOS and NLOS scenarios.

\subsection{Evaluation of EMR for different parameter settings}

Next, we evaluate the EMR metric for different $\alpha, \phi_{s}$, and $P_{\min }$ values. We change the value of one of the parameters while keeping the others fixed and repeat this procedure for all the parameters. Fig. 8(a) shows the change in the EMR with $\alpha$. As $\alpha$ is increased, weights of the weaker paths in (14) decrease and hence the EMR decreases. The EMR attains the minimum value for the measurement at TX1-RX3 for all $\alpha$ values, i.e., 2.30, 1.41, and 1.15 for $\alpha=$ $0.1, \quad \alpha=0.4$, and $\alpha=0.7$, respectively. This result can be attributed to the geometry of the environment at these particular TX-RX locations which allows only the LOS path and reflections from a glass window. Thus having an EMR close to 1 means there is only one or two paths (other than the strongest path) with relatively low power for that TXRX location and $\phi_{s}$ value. TX1-RX3 and TX2-RX12 both have the same number of clusters (see Fig. 6); however, the EMR is notably larger for the latter. Moreover, as $\alpha$ increases, the rate of decrease in the EMR for TX1-RX3 is higher than in TX2-RX12. From this observation, it can be inferred that the alternate paths for TX2-RX12 have relatively higher power and hence they are more valuable. 


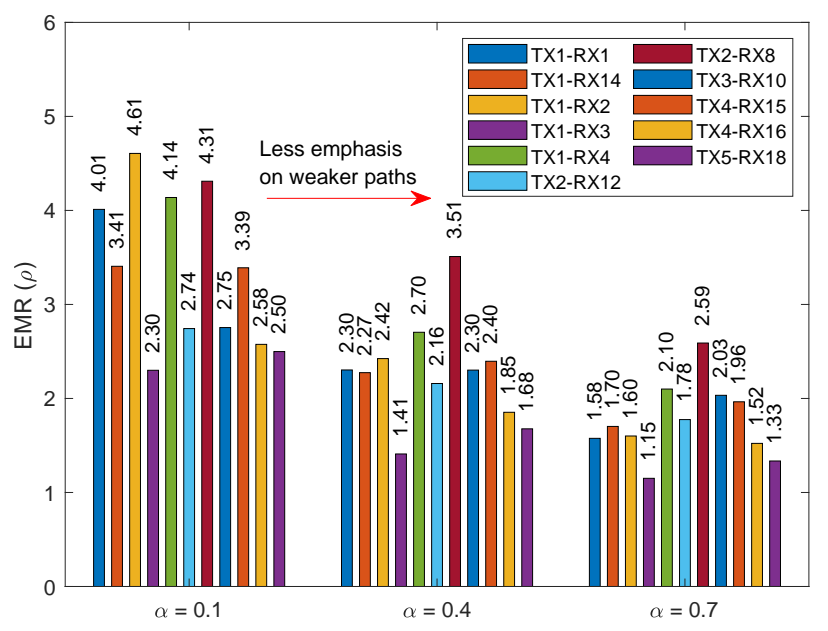

(a)

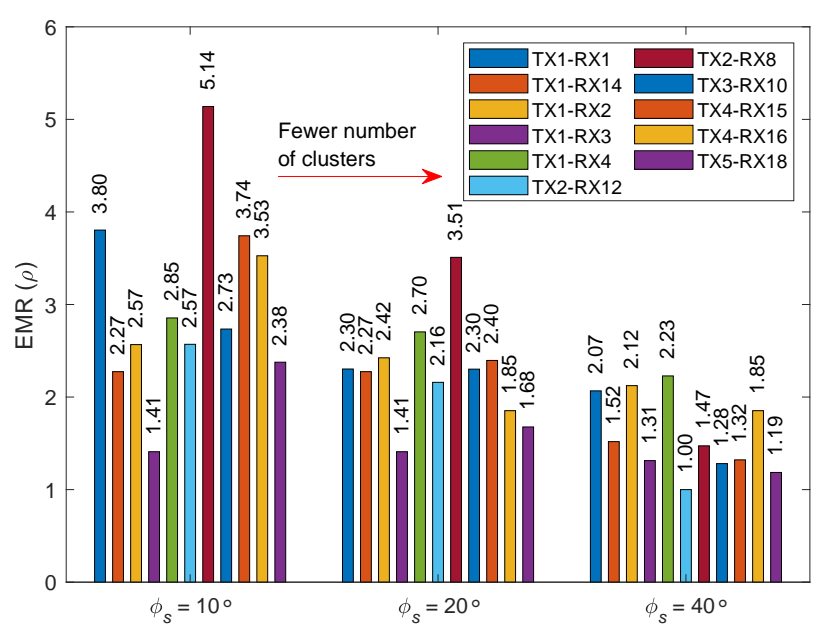

(b)

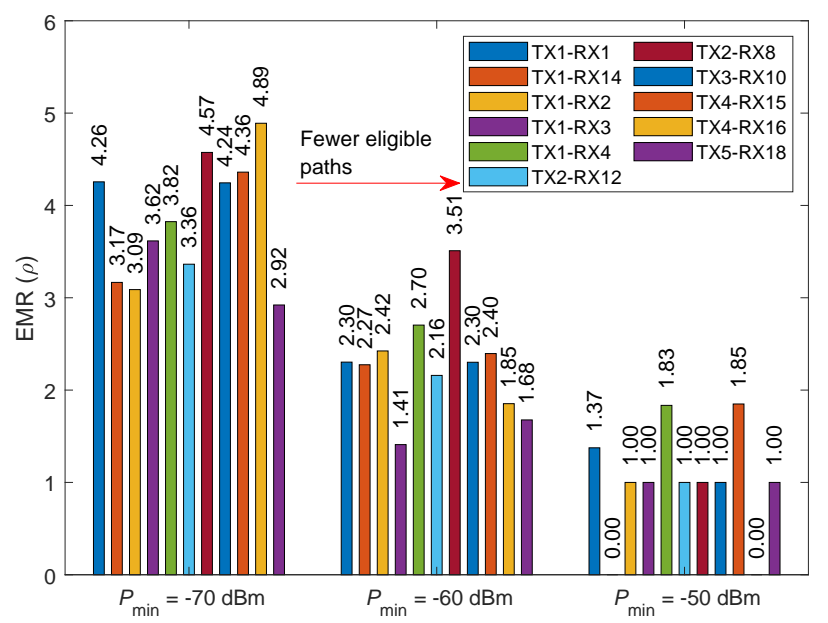

(c)

Fig. 8 - EMR for the LOS measurements for: (a) different importance factors $\alpha\left(\phi_{s}=20^{\circ}, P_{\min }=-60 \mathrm{dBm}\right)$; (b) different blockage widths $\phi_{s}\left(\alpha=0.4, P_{\min }=-60 \mathrm{dBm}\right)$; and (c) different MPC thresholds $P_{\min }$ $\left(\alpha=0.4, \phi_{s}=20^{\circ}\right)$.

Fig. 8(b) shows the impact of $\phi_{s}$ on the metric. Since larger $\phi_{s}$ values make it more difficult to satisfy the constraint in (13), there is a lower number of clusters with
Table 6 - Mean and standard deviation of EMR for different floors and different TX locations on the same floor. TX1\&TX2 are on the second floor, and TX4\&TX5 are on the fourth floor.

\begin{tabular}{lll||cl||cc}
\hline EMR & Floor 2 & Floor 4 & TX1 & TX2 & TX4 & TX5 \\
\hline mean $(\rho)$ & 1.81 & 1.66 & 1.72 & 1.98 & 2.08 & 1.24 \\
std $(\rho)$ & 1.06 & 0.55 & 0.89 & 1.5 & 0.28 & 0.38 \\
\hline
\end{tabular}

the increase in $\phi_{s}$. So fewer terms add up while calculating the EMR metric, which, in turn, results in smaller EMR values. When $\phi_{s}=40^{\circ}$, the EMR is calculated to be 1 for TX2-RX12, which means that the spatial diversity of the paths is poor. That is, there is only one effective path, and the communication can easily be severed if that path is blocked.

Finally, we look into the relation between the EMR and $P_{\text {min }}$ in Fig. 8(c). Since increasing $P_{\text {min }}$ results in fewer eligible peaks that meet the minimum power requirements, the EMR decreases. When $P_{\text {min }}$ is increased up to $-50 \mathrm{dBm}$, we observe that, for two of the measurements (i.e., TX1-RX14 and TX4-RX16), there is not a single path that has a power level above $-50 \mathrm{dBm}$, and thus the EMR becomes zero. In addition, for most of the remaining TX$\mathrm{RX}$ locations, there is only one effective path.

\subsection{Characterization of the measurement en- vironment using EMR}

In this section, we demonstrate how the EMR can be used to characterize the whole measurement environment. To this end, we give the box plots of the EMR metric in Fig. 9 for LOS and NLOS scenarios. The horizontal lines indicate (from the top) the maximum, the 75th percentile, the median (also colored red), the 25 th percentile, and the mimimum of the data. The plus signs represent the outliers. We see that the EMR values for the NLOS measurements show the same trend with the change in the parameters as in the LOS measurements. In addition, the EMR values, as expected, are smaller in the NLOS measurements than the LOS measurements. Except for the case, where $P_{\min }$ is set to $-70 \mathrm{dBm}$, the first quartile for the NLOS measurements is zero. This indicates that for some of the NLOS scenarios there is no link between the TX and the RX as mmWave frequencies are highly sensitive to blockages. However, we observe that, when $P_{\min }$ is below $-50 \mathrm{dBm}$, the third quartile of the EMR for the NLOS measurements is above 1.5 for all the $\phi_{s}$ values considered. This implies that in most of the NLOS scenarios there are at least two beam directions that can be utilized for directional communication.

As indicated earlier, the EMR can also be used to quantify the effective multipath richness at a certain part of a measurement environment or to compare the candidate BS/AP locations in terms of their resilience to blockages. To demonstrate these uses of the metric, in Table 6, we give the mean and standard deviation of the EMR calcu- 


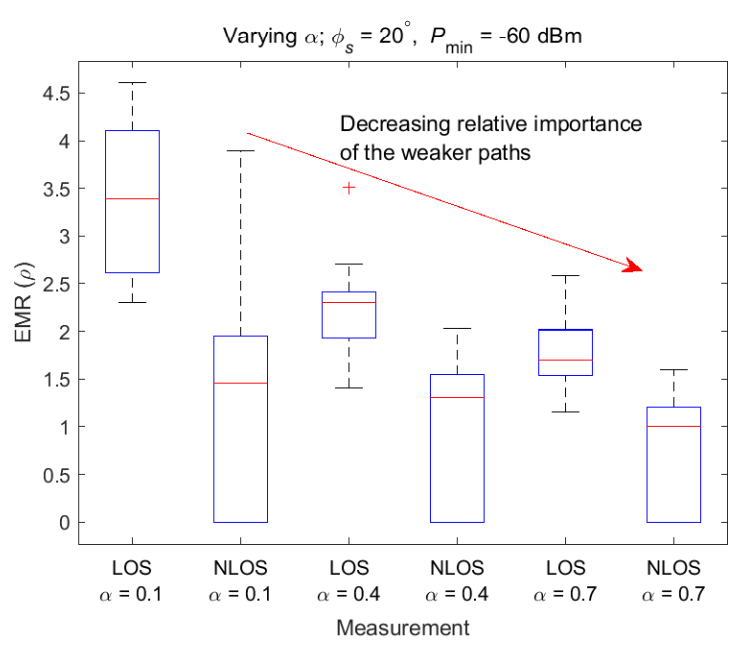

(a)

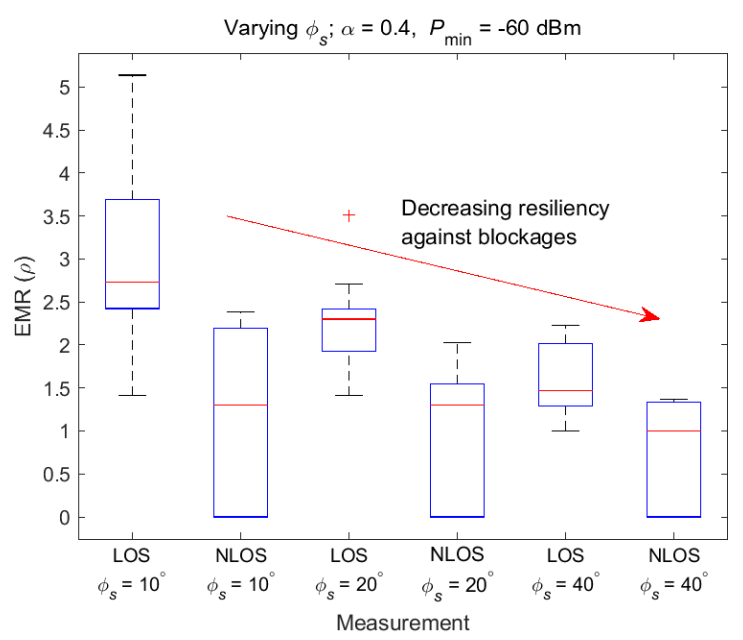

(b)

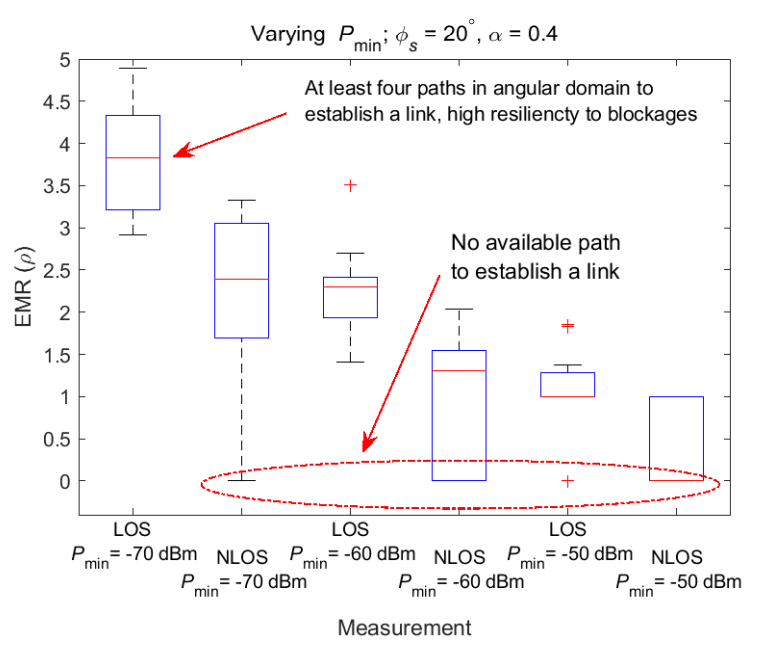

(c)

Fig. 9 - Box plots of the EMR from LOS and NLOS measurements for (a) different importance factors $\alpha\left(\phi_{s}=20^{\circ}, P_{\min }=-60 \mathrm{dBm}\right)$, (b) different blockage widths $\phi_{s}\left(\alpha=0.4, P_{\min }=-60 \mathrm{dBm}\right)$, and (c) different MPC thresholds $P_{\min }\left(\alpha=0.4, \phi_{s}=20^{\circ}\right)$.

lated for all TX-RX location pairs on the second and fourth floor of the library. We also show the same statistics for two different TX locations on the same floors. It can be observed that the second floor provides a higher mean EMR than the fourth floor but the standard deviation is also higher for the former. A similar observation can be made for the TX locations TX1 and TX2, which are placed on the second floor. Even though TX2 provides a higher EMR on the average than TX1, the standard deviation of the EMR is also higher at TX2, making it a difficult decision to choose between the two locations. However, for the TX locations TX4 and TX5 on the fourth floor, the mean EMR is higher and the standard deviation of the EMR is smaller at TX4 than at TX5. Therefore, it can be concluded that TX4 is a more preferable location than TX5. It should be noted that for a fair comparison between the EMR of different TX locations, one should perform several measurements at the same set of RX locations. Similarly, while comparing the EMR of different floors, to remove the bias in the EMR values, we suggest considering a comparable number of measurements for both LOS and NLOS scenarios on each floor.

We conclude with an important caveat. The EMR metric calculated based on the measurements at $28 \mathrm{GHz}$ cannot be extrapolated to different bands. For a given propagation environment, it would be possible to compare the EMR at two different frequencies (e.g., a mmWave and a THz frequency) only when the measurements are available for both of the frequencies. As discussed earlier in Section 1, the EMR metric depends on the carrier frequency (due to different levels of attenuation at different frequencies) along with some other parameters. This is also the reason why this metric can help to evaluate the suitability of an environment for a particular deployment band. On the other hand, the EMR is a measure of how many effective alternate paths are available and how valuable they are for establishing a reliable link. Since NLOS directional communication is critical to compensating for the high attenuation at high frequencies (i.e., mmWave and $\mathrm{THz}$ frequencies), the knowledge of the backup paths is more important at these frequencies. Therefore, this metric is especially informative for the effective deployment and operation of the network infrastructure at higher frequencies.

\section{CONCLUSION}

In this paper, we introduced a new metric to assess the effective multipath richness of a given environment, which is key to the success of directional communications in next generation wireless networks. Due to the use of higher frequencies (mmWave and THz), increased link directionality is critical to be able to maintain a reasonable communication range, and it is critical to assess the robustness of such directional links against blockages. In this context, our proposed EMR metric takes into consideration the required power level, the angular diversity of the paths, and the angular width of the blockage with respect to transmitter and receiver positions. It also allows to adjust the relative importance of the paths (directions) that are not the primary choice for communications. 
To summarize our key contribution, using a single scalar value, the proposed EMR metric provides a measure of the suitability and resilience of a specific propagation environment for directional communications under blockage, which are not captured directly by any of the other existing metrics in the literature. It is more informative than the number of clusters and RMS-DS/AS, as it provides the information on the number of alternate paths as well as the strength of the weaker paths at the same time. The metric was evaluated using real channel measurements at $28 \mathrm{GHz}$ in a library environment and shown to be useful in measuring the resilience of the propagation links to blockages. We believe that this metric will complement the other well-known propagation metrics in characterizing different mmWave propagation environments and improving the deployment and operation of next-generation mmWave networks.

\section{ACKNOWLEDGMENT}

This work has been supported in part by NASA under the Federal Award ID number NNX17AJ94A, in part by NSF through CNS-1916766, and in part by DOCOMO Innovations, Inc.

\section{REFERENCES}

[1] W. Khawaja, O. Ozdemir, Y. Yapici, F. Erden, and I. Guvenc. "Coverage Enhancement for NLOS mmWave Links Using Passive Reflectors". In: IEEE Open J. Commun. Soc. 1.1 (May 2020), pp. 263-281.

[2] D. W. Matolak, M. Mohsen, and J. Chen. "Path Loss at $5 \mathrm{GHz}$ and $31 \mathrm{GHz}$ for Two Distinct Indoor Airport Settings". In: Proc. Eur. Signal Process. Conf. (EUSIPCO). A Coruna, Spain, Sept. 2019, pp. 1-5.

[3] Ozgur Ozdemir, Fatih Erden, Ismail Guvenc, Taha Yekan, and Tom Zarian. "28 GHz mmWave Channel Measurements: A Comparison of Horn and Phased Array Antennas and Coverage Enhancement Using Passive and Active Repeaters". In: Proc. IEEE SoutheastCon. Raleigh, NC, Mar. 2020, pp. 1-6.

[4] J. Ko, Y. Cho, S. Hur, T. Kim, J. Park, A. F. Molisch, K. Haneda, M. Peter, D. Park, and D. Cho. "MillimeterWave Channel Measurements and Analysis for Statistical Spatial Channel Model in In-Building and Urban Environments at $28 \mathrm{GHz}$. In: IEEE Trans. Wireless Commun. 16.9 (Sept. 2017), pp. 58535868.

[5] Fatih Erden, Ozgur Ozdemir, Wahab Khawaja, and Ismail Guvenc. "Correction of Channel Sounding Clock Drift and Antenna Rotation Effects for mmWave Angular Profile Measurements". In: IEEE Open J. Commun. Soc. (July 2020), pp. 71-87.
[6] R. Wang, C. U. Bas, Z. Cheng, T. Choi, H. Feng, Z. Li, X. Ye, P. Tang, S. Sangodoyin, J. Gómez-Ponce, R. Monroe, T. Henige, G. Xu, J. Zhang, J. Park, and A. F. Molisch. "Enabling Super-Resolution Parameter Estimation for mm-Wave Channel Sounding". In: IEEE Trans. Wireless Commun. 19.5 (Feb. 2020), pp. 3077-3090.

[7] P. B. Papazian, J. Choi, J. Senic, P. Jeavons, C. Gentile, N. Golmie, R. Sun, D. Novotny, and K. A. Remley. "Calibration of millimeter-wave channel sounders for super-resolution multipath component extraction". In: Proc. Eur. Conf. Antennas Propag. (EuCAP). Davos, Switzerland, Apr. 2016, pp. 1-5.

[8] X. Wu, C. Wang, J. Sun, J. Huang, R. Feng, Y. Yang, and X. Ge. "60-GHz Millimeter-Wave Channel Measurements and Modeling for Indoor Office Environments". In: IEEE Trans. Antennas Propag. 65.4 (Sept. 2017), pp. 1912-1924.

[9] $\mathrm{K} \mathrm{Yu}, \mathrm{Q} \mathrm{Li}$, and M Ho. "Measurement investigation of tap and cluster angular spreads at $5.2 \mathrm{GHz}$. In: IEEE Trans. Antennas Propag. 53.7 (July 2005), pp. 2156-2160.

[10] Zhicheng Yang, Parth H. Pathak, Jianli Pan, Mo Sha, and Prasant Mohapatra. "Sense and deploy: Blockage-aware deployment of reliable $60 \mathrm{GHz}$ mmWave WLANs". In: Proc. IEEE Int. Conf. Mobile Ad Hoc Sensor Syst. (MASS). Chengdu, China, Oct. 2018, pp. 397-405.

[11] Greg Durgin and TS Rappaport. "Basic relationship between multipath angular spread and narrowband fading in wireless channels". In: Electron. Lett. 34.25 (Dec. 1998), pp. 2431-2432.

[12] Y. Chen, W. Cheng, and L. Wang. "Learning-assisted beam search for indoor mmWave networks". In: Proc. IEEE Wireless Commun. Netw. Conf. Workshops (WCNCW). Barcelona, Spain, Apr. 2018, pp. 320-325.

[13] I. Mavromatis, A. Tassi, R. J. Piechocki, and A. Nix. "Efficient Millimeter-Wave Infrastructure Placement for City-Scale ITS". In: Proc. IEEE Veh. Technol. Conf. (VTC). Kuala Lumpur, Malaysia, June 2019, pp. 1-5.

[14] Marco Giordani, Michele Polese, Arnab Roy, Douglas Castor, and Michele Zorzi. "A tutorial on beam management for 3GPP NR at mmWave frequencies". In: IEEE Commun. Surveys Tuts. 21.1 (Sept. 2018), pp. 173-196.

[15] C. Huang, R. He, Z. Zhong, Y. Geng, Q. Li, and Z. Zhong. "A Novel Tracking-Based Multipath Component Clustering Algorithm". In: IEEE Antennas Wireless Propag. Lett. 16 (Aug. 2017), pp. 26792683. 
[16] T. S. Rappaport, G. R. MacCartney, M. K. Samimi, and S. Sun. "Wideband Millimeter-Wave Propagation Measurements and Channel Models for Future Wireless Communication System Design". In: IEEE Trans. Commun. 63.9 (Sept. 2015), pp. 3029-3056.

[17] Fatih Erden, Ozgur Ozdemir, and Ismail Guvenc. "28 GHz mmWave Channel Measurements and Modeling in a Library Environment". In: Proc. IEEE Radio Wireless Symp. (RWS). San Antonio, TX, Jan. 2020, pp. 52-55.

\section{AUTHORS}

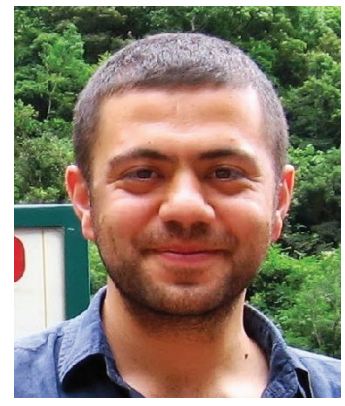

Fatih Erden received B.S. and M.S. degrees from Bilkent University, Ankara, Turkey, in 2007 and 2009, respectively, and a Ph.D. degree from Hacettepe University, Ankara, Turkey, in 2015, all in electrical and electronics engineering. From 2015 to 2016, he was an assistant professor with the Department of Electrical and Electronics Engineering, Atilim University, Ankara, Turkey. From 2016 to 2018, he was with the Signal Processing Group at Bilkent University, Ankara, Turkey as a postdoctoral researcher. Since 2018, he has been a research associate at the Department of Electrical and Computer Engineering at North Carolina State University. His research interests include signal and image processing, time-series analysis, machine learning in communications, mmWave communications, and UAVs. Dr. Erden received the Scientific and Technological Research Council of Turkey National M.S. Scholarship Award in 2007. He received the Best Paper Award in the Journal of Modern Power Systems and Clean Energy in 2019.

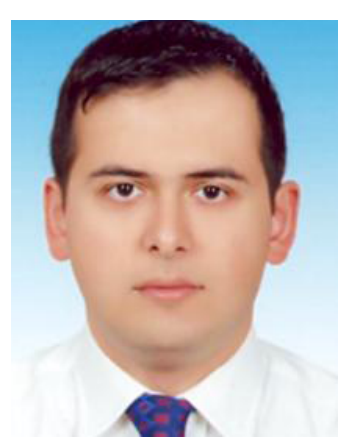

Ozgur Ozdemir received BS degree in electrical and electronics engineering from Bogazici University, Istanbul, Turkey, in 1999 and MS and PhD degrees in electrical engineering from University of Texas at Dallas, Richardson, TX, USA, in 2002 and 2007, respectively. Before joining the Department of Electrical and Computer Engineering at NCSU as a visiting research scholar, he has been an assistant professor at the Department of Electrical and Electronics Engineering, Fatih University, Turkey and a postdoctoral scholar at Qatar University, Doha, Qatar. His research interests include opportunistic approaches in wireless systems, experimental multiple-antenna systems, digital compensation of radio-frequency impairments, wireless multicarrier communications, and software defined radios.

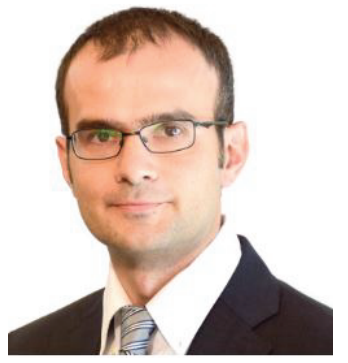

Ismail Guvenc received his Ph.D. degree in electrical engineering from the University of South Florida in 2006. He was with Mitsubishi Electric Research Labs during 2005, with DOCOMO Innovations between 2006-2012, and with Florida International University between 2012-2016. Since 2016,

he has been an associate professor with the Department of Electrical and Computer Engineering at North Carolina State University. His recent research interests include $5 \mathrm{G}$ wireless systems, communications and networking with drones, and heterogeneous wireless networks. He has published more than 200 conference/journal papers and book chapters, and several standardization contributions. He co-authored/co-edited three books for Cambridge University Press, served as an editor for IEEE Communications Letters (2010-2015), IEEE Wireless Communications Letters (2011-2016), and IEEE Transactions on Wireless Communications (2016-present), and as a guest editor for several other journals. Dr. Guvenc is an inventor/coinventor in some 30 U.S. patents and he is a senior member of the National Academy of Inventors. He is a recipient of the NCSU ECE R. Ray Bennett Faculty Fellow Award (2019), FIU College of Engineering Faculty Research Award (2016), NSF CAREER Award (2015), Ralph E. Powe Junior Faculty Enhancement Award (2014), and USF Outstanding Dissertation Award (2006).

David W. Matolak received B.S. degree in electrical engineering from Pennsylvania State University, State College, PA, USA, in 1983, an M.S. degree in electrical engineering from the University of Massachusetts, Boston, MA, USA, in 1987, and a Ph.D. degree in electrical engineering from the University of Virginia, Charlottesville, VA, USA, in 1995. He has over 25 years' experience in communication system research, development, and deployment, with industry, government institutions, and academia, including AT\&T Bell Labs, Murray Hill, NY, USA; L3 Communication Systems, New York NY, USA; MITRE, McLean, VA, USA; and Lockheed Martin, Bethesda, MD, USA. He was a professor with Ohio University, Athens, OH, USA, from 1999 to 2012. Since 2012, he has been a professor with the University of South Carolina, Columbia, SC, USA. He has over 250 publications and eight patents. His research interests are radio channel modeling and communication techniques for non-stationary fading channels. Dr. Matolak has organized multiple IEEE workshops and special sessions, and is an associate editor of the IEEE TRANSACTIONS ON WIRELESS COMMUNICATIONS. He is also a member of standards groups in RTCA and ITU, and a member of Eta Kappa Nu, Sigma Xi, Tau Beta Pi, URSI, ASEE, and AIAA. 